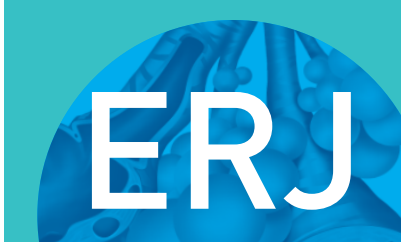

open research

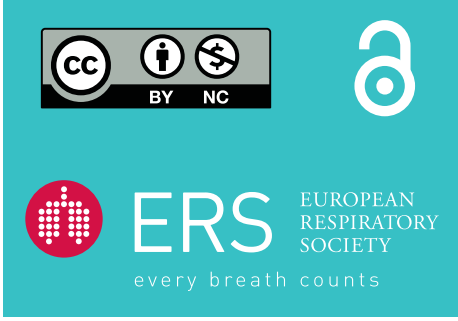

\section{Microparticles in COVID-19 as a link between lung injury extension and thrombosis}

\section{To the Editor:}

Among the distinctive features of coronavirus disease 2019 (COVID-19), numerous reports have stressed the importance of vascular damage associated with coagulopathy onset [1]. Histological analysis of pulmonary vessels in patients with COVID-19 revealed severe endothelial injury associated with intracellular severe acute respiratory syndrome coronavirus 2 (SARS-CoV-2) virus and disrupted endothelial cell membranes together with widespread thrombosis and occlusion of alveolar capillaries. Microparticles (MPs) shed by apoptotic/stimulated cells of various cellular lineages, including platelets, leukocytes, macrophages or endothelial cells, are reliable markers of vascular damage [2] released upon pro-inflammatory conditions and behave as active participants in the early steps of clot formation [3]. Circulating MPs promote procoagulant responses due to the exposure of tissue factor, the physiological activator of the coagulation cascade, and of negatively charged phospholipids, such as phosphatidylserine, required for the assembly of the tenase and prothrombinase coagulation complexes ultimately leading to thrombin generation, through which they can precisely be quantified [4]. MPs carry angiotensinconverting enzyme (ACE)1 and upregulate ACE1 expression in neighbouring endothelial cells [5]. By contrast, exosomes were recently reported to convey ACE2, the cell-entry receptor for SARS-CoV-2 [4], in the vasculature [6]. ACE2 converts angiotensin II (Ang II) into angiotensin 1-7 (Ang 1-7), which by virtue of its actions on the Mas receptor, limits the noxious effects of Ang II. Pioneering data have demonstrated that the renin-angiotensin system has a crucial role in severe acute injury and that ACE2 has a protective role in acute lung injury mediated by SARS-CoV [7]. According to this paradigm, the loss of ACE2 function following binding by SARS-CoV-2 may contribute to unopposed Ang II accumulation that further exacerbates tissue injury and promotes inflammation, MPs release and thrombosis. During SARS-CoV-2 infection, we hypothesised that various factors including inflammatory burden, Ang II, altered shear stress and hypoxic vasoconstriction, could enhance MPs shedding by various cell lineages including the alveolar vascular endothelium and contribute to clot formation.

We identified consecutive COVID-19 patients admitted to the intensive care unit (ICU) and non-ICU units at Strasbourg University Hospital from 25 February 2020 to 19 April 2020. Blood samples were obtained in the $48 \mathrm{~h}$ following admission. Patients were eligible if lupus anticoagulant (LA) was positively detected and remaining plasma available. To ascertain their contribution in COVID-19 lung injuries and related coagulopathy, procoagulant MPs levels, pro-inflammatory cytokines (interleukin-6 (IL-6), monocyte chemotactic protein-1 (MCP-1)), and cyto-adhesins (intercellular adhesion molecule 1 (ICAM-1), vascular cell adhesion molecule 1 (VCAM-1)), as markers of endothelium damage, were measured in 52 patients with COVID-19 infection and positive LA. In addition, leukocytes, platelets, fibrinogen, activated partial thromboplastin time (APTT), von Willebrand factor antigen (vWF:Ag) and D-dimers levels were determined. Control groups were age-matched and consisted of 40 non-COVID-19 patients with positive LA screened at the Haematology department for acquired coagulopathy and 20 healthy volunteers (blood donors) without any cardiovascular risk factors or significant comorbidities.

@ERSpublications

Procoagulant microparticles are associated with the extent of lung injuries in \#COVID19 and pulmonary thrombosis https://bit.ly/3eX2LPc

Cite this article as: Morel O, Marchandot B, Jesel L, et al. Microparticles in COVID-19 as a link between lung injury extension and thrombosis. ERJ Open Res 2021; 7: 00954-2020.DOC [https:// doi.org/10.1183/23120541.00954-2020].

Copyright (CThe authors 2021. This version is distributed under the terms of the Creative Commons Attribution NonCommercial Licence 4.0. For commercial reproduction rights and permissions contact permissions@ersnet.org 
Measurement of MPs procoagulant activity was performed with the ZYMUPHEN MP-Activity ELISA kit (Hyphen Biomed, Neuville-sur-Oise, France). MPs were captured on immobilised annexin V and their phosphatidylserine content measured by a prothrombinase assay using a microtitration plate reader equipped with kinetics software. In this assay, blood clotting factors (FXa, FVa, FII) and calcium concentrations were determined to ensure that phosphatidylserine is the rate-limiting parameter in the generation of soluble thrombin from prothrombin. Results were expressed as nanomolar phosphatidylserine equivalent (nmol. $\mathrm{L}^{-1}$ PhtdSerEq) by reference to a standard curve constructed with liposomes of known composition and concentration. With respect to characterisation of MPs by flow cytometry, this functional method allows the determination of MPs prothrombotic properties [4, 8-10].

Haemostasis assays (fibrinogen, vWF:Ag, D-dimer, LA detection) were analysed on STA-R Evolution (Diagnostica Stago, Asnières-sur-Seine, France) with standard commercial reagents and protocols.

Two screening tests were performed: a diluted Russell viper venom time (dRVVT screen) made with the STA-Staclot dRVV screen reagent (Stago), and an activated partial prothromboplastin time (APPT) performed with the STA-PPT A reagent (Stago). Positivity of one or both screening tests induced a mixing test at 1:1 proportion with a commercial frozen pooled normal plasma (PNP) (Cryocheck Cryopep, Montpellier, France). Moreover, a positive dRVVT screen induced a confirmatory test with an increased concentration of phospholipids (dRVVT confirm), performed with the STA-Staclot dRVV Confirm reagent (Stago). dRVVT screen, dRVVT confirm and APTT results were expressed as a ratio of patient-to-PNP. Mixing tests results were expressed as an index of circulating anticoagulant. LA was considered positive only if the normalised dRVVT ratio (screen ratio/confirm ratio) was $>1.2$ and all causes of false positive were excluded (i.e. anticoagulation conditions).

MCP-1, IL-6, VCAM-1 and ICAM-1 concentrations were determined with a Quantikine ELISA kit (Research and Diagnostic Systems, Minneapolis, MN, USA).

The extent of COVID-19 disease on chest computed tomography (CT) was assessed as extension of pulmonary injuries as a percentage of the total lung parenchyma and classified according to the European Society of Radiology/European Society of Thoracic Imaging guidelines as minimal (stage $1<10 \%$ ), moderate (stage $2=10-25 \%$ ), severe (stage $3=25-50 \%$ ) and critical (stage $4>50 \%$ ). Patients with suspected acute pulmonary embolism, based on their clinical, echocardiographic or laboratory parameters had a CT pulmonary angiography performed, either at the admission or during their stay. The present study was approved by the research ethics committee of Strasbourg Hospital and written informed consent was obtained from all patients.

Among the 52 patients with COVID-19 infection and positive LA (mean age $54 \pm 20$ years; male sex 78.8\%), 12 patients experienced acute pulmonary embolism and 20 showed severe lung injury severity ( $>50 \%)$ assessed by chest CT. No baseline differences between COVID-19 patients and non-COVID-19 patients with positive LA could be identified apart from obesity $(18(34.6 \%)$ versus $3(7.5 \%), \mathrm{p}=0.002)$ and smoking habits $(2(3.8 \%)$ versus $7(17.5 \%), \mathrm{p}=0.029)$.

A stepwise increase in pro-inflammatory cytokines and cyto-adhesins levels could be seen among the three groups, with highest values measured in COVID-19 patients (figure 1a-d). Procoagulant MPs were 2.5 times higher in patients with LA as compared with healthy volunteers (5.7 (3.3-8.7) nM PhtdSerEq) and with no further increase between COVID-19 and non-COVID-19 patients (13.2 (6.7-21.4) versus 13.0 (7.5-20.3) $\mathrm{nM}$ PhtdSerEq) (figure 1e). Index of circulating anticoagulant was equivalent between non-COVID-19 and COVID-19 patients (1.41 (1.35-1.58) versus 1.38 (1.27-1.49); $\mathrm{p}=0.233)$. No impact of obesity on MPs release or cytokines and cyto-adhesins levels could be established. The highest levels of procoagulant MPs could be seen in patients with pulmonary embolism (20.6 (12.1-27.1) versus 10.1 (5.6-18.8) nM PhtdSerEq; $\mathrm{p}=0.003$ ) (figure 1f) or those with severe lung injuries (19.8 (11.0-27.4) versus 8.1 (4.6-17.8) nM PhtdSerEq; p=0.004) (figure 1g). By multivariable analysis, MPs were the sole independent predictor of critical lung injury $(4.06,95 \% \mathrm{CI}$ $(1.10-14.93) ; \mathrm{p}=0.035)$.

In the past, several reports have emphasised that circulating endothelial MPs are a reliable marker of early lung destruction in cigarette smokers [11], pulmonary hypertension [12] and various lung diseases [13]. More recently, a study performed in microvascular lung endothelial cells and in patients with acute respiratory distress syndrome has emphasised the importance of ACE-bearing MPs as an important marker of disease severity [14]. Consistent with this paradigm, we report that procoagulant MPs are associated with the extent of lung injuries in COVID-19 and pulmonary thrombosis. Additional work is needed to confirm that MPs behave as bioactive shuttles spreading the thrombotic burden within the vasculature in SARS-CoV-2 infection. 

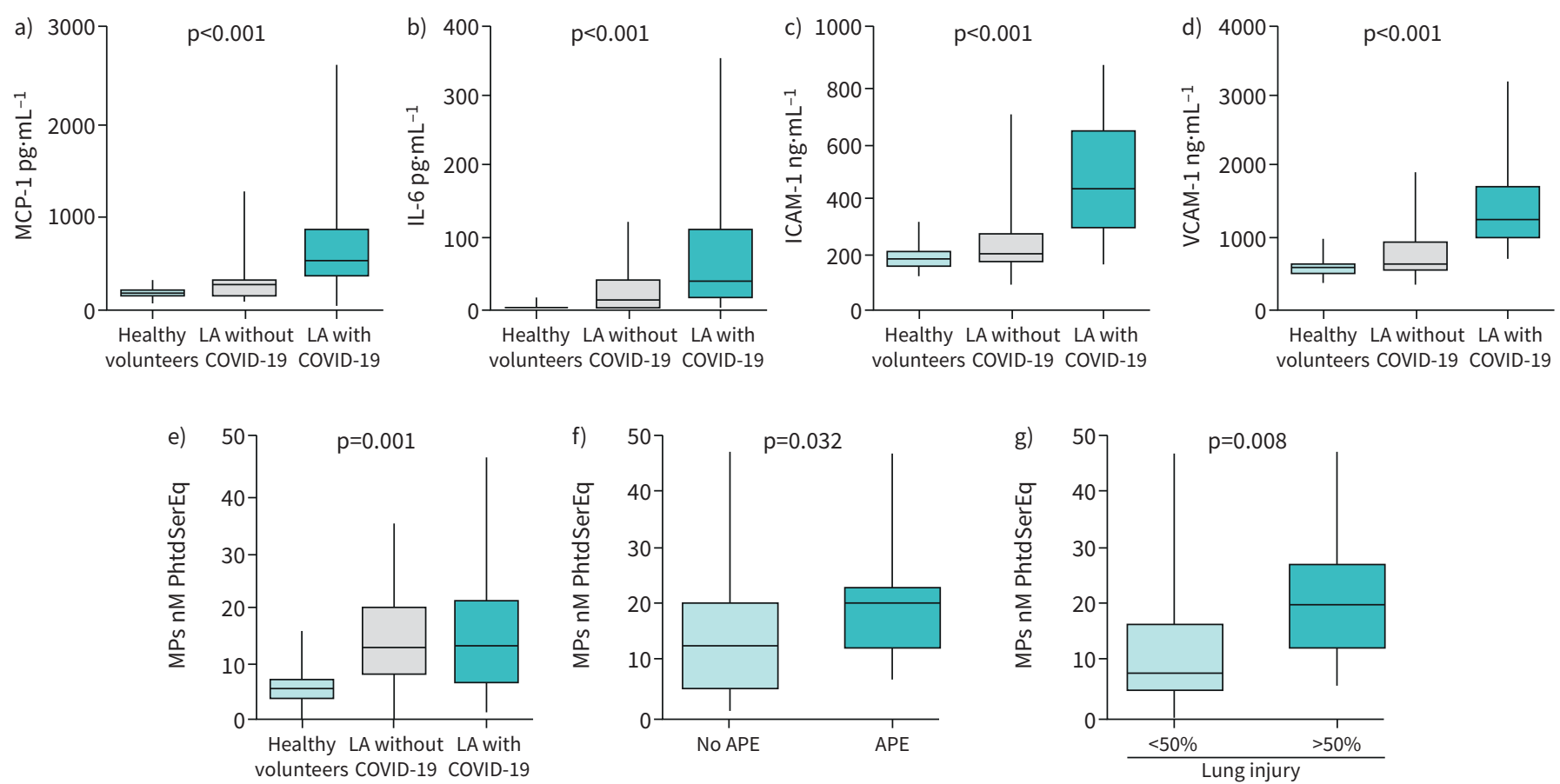

FIGURE 1 a, b) Pro-inflammatory cytokines (monocyte chemoattractant protein-1 (MCP-1) and interleukin (IL)-6) and c, d) cyto-adhesins (intercellular adhesion molecule-1 (ICAM-1) and vascular cell adhesion molecule-1 (VCAM-1)) levels in the study population. e) Procoagulant microparticles (MPs) levels in the study population. f) Procoagulant MPs levels in COVID-19 patients stratified by concomitant in-hospital acute pulmonary embolism (APE). g) Procoagulant MPs levels in COVID-19 patients stratified by lung injury severity (critical $>50 \%$ ) assessed by computed tomography. Boxes indicate median and 25-75th (interquartile) range. Bars indicate minimum and maximum values. Comparisons between groups were made using nonparametric tests. LA: lupus anticoagulant; PhtdSerEq: phosphatidylserine equivalent.

Olivier Morel $\oplus^{1,2}$, Benjamin Marchandot $\odot^{1}$, Laurence Jesel ${ }^{1,2}$, Laurent Sattler ${ }^{3}$, Antonin Trimaille ${ }^{1}$, Anais Curtiaud ${ }^{1}$, Mickael Ohana ${ }^{4}$, Samira Fafi-Kremer ${ }^{5}$, Valerie Schini-Kerth ${ }^{2}$, Lelia Grunebaum ${ }^{3}$ and Jean-Marie Freyssinet ${ }^{3}$

${ }^{1}$ Université de Strasbourg, Faculté de Médecine, Pôle d’Activité Médico-Chirurgicale Cardio-Vasculaire, Nouvel Hôpital Civil, Centre Hospitalier Universitaire, Strasbourg, France. ${ }^{2}$ UMR INSERM 1260, Regenerative Nanomedicine, Faculté de Pharmacie, Université de Strasbourg, Illkirch, France. ${ }^{3}$ Université de Strasbourg, Pôle de Biologie, Département d’Hémostase, Centre Hospitalier Universitaire, Strasbourg, France. ${ }^{4}$ Université de Strasbourg, Département de Radiologie, Centre Hospitalier Universitaire, Strasbourg, France. ${ }^{5}$ Université de Strasbourg, Pôle de Biologie, Département de Virologie, Centre Hospitalier Universitaire, Strasbourg, France.

Correspondence: Olivier Morel, Pôle d'Activité Médico-Chirurgicale Cardiovasculaire, Nouvel Hôpital Civil, BP 426-67091 Strasbourg, France. E-mail: olivier.morel@chru-strasbourg.fr

Received: 18 Dec 2020 | Accepted: 8 April 2021

Author contributions: O. Morel: study conception and design, data collection and interpretation, drafting of the manuscript, and critical revision for important intellectual content. B. Marchandot: drafting of the manuscript and editing. L. Jesel: Study conception and design, drafting of the manuscript and critical revision for important intellectual content. L. Sattler, A. Trimaille, A. Curtiaud and M. Ohana: Data collection and interpretation. S. Fafi-Kremer: Drafting of the manuscript, data collection and interpretation. V. Schini-Kerth and L. Grunebaum: Data collection and interpretation, critical revision for important intellectual content. J.-M. Freyssinet: Drafting of the manuscript, critical revision for important intellectual content and supervision.

Conflict of interest: O. Morel has nothing to disclose. B. Marchandot has nothing to disclose. L. Jesel has nothing to disclose. L. Sattler has nothing to disclose. A. Trimaille has nothing to disclose. A. Curtiaud has nothing to disclose. M. Ohana has nothing to disclose. S. Fafi-Kremer has nothing to disclose. V. Schini-Kerth has nothing to disclose. L. Grunebaum has nothing to disclose. J-M. Freyssinet reports other (royalties) from HYPHEN BioMed, during the conduct of the study; In addition, Dr. Freyssinet has a patent WO1996003655A1 with royalties paid to HYPHEN BioMed and Patent WO1996003655A1 expired several years ago, in 2013-2014 as far as it can be remembered, when giving up paying for yearly fees no longer covered by royalties from HYPHEN BioMed for their Zymuphen MP-activity 
kits. For instance, patent fee for 2012 was 1902.83 EUR for France and Germany only, not including Japan and US rights. Last royalties, over 36 months (3 years) amounted to 5153 EUR, submitted to $16 \%$ taxes paid to the French tax system

Support Statement: This work was supported by the Groupe pour l'Enseignement de la Recherche Cardio-vasculaire en Alsace, France, by Fondation Coeur et Recherche and by an unrestricted research grant from Boehringer Ingelheim Pharma GmbH \& Co. KG, Biberach, Germany. Funding information for this article has been deposited with the Crossref Funder Registry.

\section{References}

1 Ackermann M, Verleden SE, Kuehnel M, et al. Pulmonary vascular endothelialitis, thrombosis, and angiogenesis in Covid-19. N Engl J Med 2020; 383: 120-128.

2 Nieri D, Neri T, Petrini S, et al. Cell-derived microparticles and the lung. Eur Respir Rev 2016; 25: 266-277.

3 Rautou PE, Vion AC, Amabile N, et al. Microparticles, vascular function, and atherothrombosis. Circ Res 2011; 109: 593-606.

4 Aupeix K, Hugel B, Martin T, et al. The significance of shed membrane particles during programmed cell death in vitro, and in vivo, in HIV-1 infection. J Clin Invest 1997; 99: 1546-1554.

5 Abbas M, Jesel L, Auger C, et al. Endothelial microparticles from acute coronary syndrome patients induce premature coronary artery endothelial cell aging and thrombogenicity: role of the Ang II/AT1 receptor/NADPH oxidase-mediated activation of MAPKs and PI3-Kinase pathways. Circulation 2017; 135: 280-296.

6 Wang J, Chen S, Bihl J. Exosome-mediated transfer of ACE2 (angiotensin-converting enzyme 2) from endothelial progenitor cells promotes survival and function of endothelial cell. Oxid Med Cell Longev 2020; 2020: 4213541.

7 Kuba K, Imai $\mathrm{Y}$, Rao S, et al. A crucial role of angiotensin converting enzyme 2 (ACE2) in SARS coronavirus-induced lung injury. Nat Med 2005; 11: 875-879.

8 Morel O, Jesel L, Hugel B, et al. Protective effects of vitamin C on endothelium damage and platelet activation during myocardial infarction in patients with sustained generation of circulating microparticles. J Thromb Haemost 2003; 1: 171-177.

9 Mallat Z, Benamer H, Hugel B, et al. Elevated levels of shed membrane microparticles with procoagulant potential in the peripheral circulating blood of patients with acute coronary syndromes. Circulation 2000; 101: 841-843.

10 Rossig L, Haendeler J, Mallat Z, et al. Congestive heart failure induces endothelial cell apoptosis: protective role of carvedilol. J Am Coll Cardiol 2000; 36: 2081-2089.

11 Gordon C, Gudi K, Krause A, et al. Circulating endothelial microparticles as a measure of early lung destruction in cigarette smokers. Am J Respir Crit Care Med 2011; 184: 224-232.

12 Bakouboula B, Morel O, Faure A, et al. Procoagulant membrane microparticles correlate with the severity of pulmonary arterial hypertension. Am J Respir Crit Care Med 2008; 177: 536-543.

13 Kubo H. Extracellular vesicles in lung disease. Chest 2018; 153: 210-216.

14 Takei Y, Yamada M, Saito K, et al. Increase in circulating ACE-positive endothelial microparticles during acute lung injury. Eur Respir J 2019; 54: 1801188. 